\title{
Fabrication and Characterization of PVDF with an Additive of Nanozeolite via Electrospinning and Non-solvent Induced Phase Separation (NIPS) Process
}

\author{
HindunNofitri Da Conceicao Isya ${ }^{1}$,Imelda Valadares Marcal $^{1}$ and Ruth R. Aquino ${ }^{1, *}$ \\ ${ }^{1}$ Mapúa University, School of Chemical, Biological, and Materials Engineering and Sciences, 658 Muralla St., Intramuros Manila, 1002 \\ Philippines
}

\begin{abstract}
In this study, polyvinylidene fluoride with an additive of nanozeolite (PVDF/NZ) membranes were prepared, characterized and evaluated. The concentrations of the nanozeolite incorporated into PVDF were varied from $0.25 \%, 0.50 \%$ and $0.75 \%$ with $\mathrm{N}$-methyl-2-pyrrolidone (NMP) as solvent and the corresponding effects of nanozeolite on the polymer matrix were investigated in terms of performance and properties. There are two methods in preparing the membranes, namely: Non-solvent Induced Phase Separation (NIPS) and electrospinning. The hydrophobicity of the membranes was characterized by contact angle, the surface morphology using Scanning Electron Microscopy (SEM), and the mechanical properties by Universal Testing Machine (UTM). The presence of organic and inorganic matter was investigated using Fourier-Transform Infrared (FTIR). The SEM images of both fabricated nanocomposite membranes showed that after the addition of nanozeolite particles into PVDF matrix has affected the surface morphology, flatsheet resulted decreasing in porous and electrospun resulted less beads and increasing fiber diameter after adding an extra amount of nanozeolite. The chemical bond or molecular structure of flat-sheet and electrospun membranes obtained same functional groups, however the electrospun resulted a high absorption of alkanes. The contact angle of both nanocomposite fabricated membranes exhibited an increasing contact angle, yet the PVDF/0.75NZ of electrospun membrane obtained higher hydrophobic surface compared to others. The result of UTM showed that on flat-sheet, the tensile strength was obtained by pure PVDF membrane while the PVDF/0.25NZ of electrospun membrane was able to achieve an optimum tensile strength. In fact, the tensile strength via NIPS need to be improved.
\end{abstract}

\section{Introduction}

Polymers are widely used as advanced materials, which are found almost in every material used in our daily life. Polyvinylidene fluoride (PVDF), due to its excellent chemical stability, thermal stability, resistance to radiation and good mechanical strength, has been widely used as a very outstanding membrane material. This polymer membrane has been employed in a large range of applications as an effective and energy-saving technique [1]. Meanwhile, Zeolite is a mesoporous material which has ability to absorbing water from harsh environment [2] due to their properties is often used as a catalyst for a purpose of water and air purification, and used extensively in various industrial applications.

For this purpose, this paper will fabricate two typesof different membrane by taking the PVDF as a base of membrane with nanozeolite as an additive and $\mathrm{N}$ Methyl-2-pyrrolidone (NMP) as a solvent. Through the comparison of two different methods will enhance the investigation and differentiate a significant change in the membrane.
This study aims to determine and compare which fabricated membrane is efficient in terms of surface morphology, tensile strength and interaction of water into surface of membrane. This study will be limited to the proposal for the various applications of membranes.

\section{Methodology}

The materials use are the media bottles, Teflon tape, stirrer, adhesive tape, A4-size smooth glass panels, long rectangular plastic basin, delicate task wipes (Kimberly Clark Kimwipes ${ }^{\mathrm{TM}}$ ), membrane support and a casting knife. The PVDF-NZ/NMP solution was prepared by varying the concentration of PVDF and nanozeolite and constant number for NMP with the ratio of 21:0:79, 20.75:0.25:79, 20.50:0.5:79 and 20.25:0.75:79.The performed experiment was done in the fume hood. For the Pure PVDF sample, the weighed PVDF pellets were dissolved in NMP with continuous magnetic stirring at $60^{\circ} \mathrm{C}$ in the hot plate until it homogenized. For PVDF/0.25NZ, PVDF/0.50NZ and PVDF/0.75NZ samples, the total percent weight of NMP was divided by $75 \%$ for the PVDF/NMP and $25 \%$ for the

*Corresponding author: rraquino@mapua.edu.ph 
Nanozeolite/NMP. The PVDF/NMP solution was heated in a $60^{\circ} \mathrm{C}$ hot plate and underwent magnetic stirring for 3 hours or more until all pellets were dissolved and achieved homogeneity.On the other hand, the Nanozeolite/NMP solution was mixed in media bottle using ultrasonicator for 2 hours to prevent agglomerations. Afterwards, the clay-NMP solutions was poured into corresponding homogenous PVDF/NMP solution with continuous magnetic stirring for another 2 hours at $40^{\circ} \mathrm{C}$ in the hot plate. Degassing was done by opening the bottle cap halfway for 2 hours or until no bubbles were seeing in the solution.

\subsection{Fabricated electrospunmembrane via electrospinning}

The PVDF-NZ/NMP polymer solution was electrospun under the parameters of $20 \mathrm{kV}$ and $150 \mathrm{~mm}$ of the distance tip-to-collector. The spinneret used in electrospinning was a $10-\mathrm{mL}$ syringe pump with $21 \mathrm{G}$ needle and $23{ }^{\circ} \mathrm{C}$ of temperature. For each sample, $4 \mathrm{~mL}$ of the solution was used electrospun with the feed rate of $1 \mathrm{~mL}$ per hour and aluminium foil as collector.

\subsection{Fabrication of flat-sheet membrane via NIPS}

A moderate amount of the PVDF-NZ/NMP solution was poured on the topmost side of the support. The casting knife with $300 \mu \mathrm{m}$ thickness was used, it was slide from the top, where the solution poured down to the bottom with uniform pressure. Then, it was immediately immersed into water bath in a careful manner to prevent any vibration and physical motion/disturbances in the water. The membrane was removed from the water after 5 minutes and air-dried overnight.

\section{Results and Discussion}

\subsection{Surface and cross-section morphology}

As shown in the fig. 1 and fig. 2, the pure PVDF formed spherulite-structure with few pinholes, after adding $0.25 \%$ of nanozeolite the surface tended to form spongy-like structure, meanwhile the spherulite-structure tended to be smothered with very few porous on the surface upon adding PVDF/0.50NZ and reduced in porous after the presence of $0.75 \%$ of nanozeolite in membrane.The results shown that after adding nanozeolite into polymer solution, the porous tend to form due to the higher viscosity of the solution. In contrary, agglomeration of particle could happen wherein the porous would tend to diminish and became smother upon adding excessive amount of nanozeolite.From the cross- sectional view in figure 2,asymmetrical spongy-like structure with fingerlike at bottom is formed after adding $0.25 \%$ of nanozeolite, however, the finger-like formed would become longer after adding $0.75 \%$ of nanozeolite.Therefore, as increasing the amount of nanozeolite and decreasing the amount of PVDF, the voids tend to appear which can increase the porous in

membranes due to the higher viscosity of the solution after adding nanozeolite.

As observed in fig. 3 and fig. 4, the number of spherical beads were seen in pure PVDF. However, the shape and size of bead-on-string were changed and less in PVDF/0.25NZ, PVDF/0.50NZ and PVDF/0.75NZ. The beads formation has been reported due to charge density, low molecular weight, low concentration and low viscosity of the polymer solution.An average fiber diameter of pure PVDF was $57.6 \pm 27.1 \mathrm{~nm}$. ThePVDF/0.25NZ was brought a dramatic change to the membrane morphology, it produced much aligned nanofibers with an increase in diameter of $105 \pm 68 \mathrm{~nm}$ and PVDF/0.50NZ solution it slightly decreased with an average fiber diameter of $83.7 \pm 44.3 \mathrm{~nm}$. On the contrary, an epic change was observed in PVDF/0.75NZ with an enormous diameter of $215.25 \pm 171.75 \mathrm{~nm}$. This phenomenon was affected by mildly increasing of nanozeolite filler into polymer solution which associated with solution viscosity.

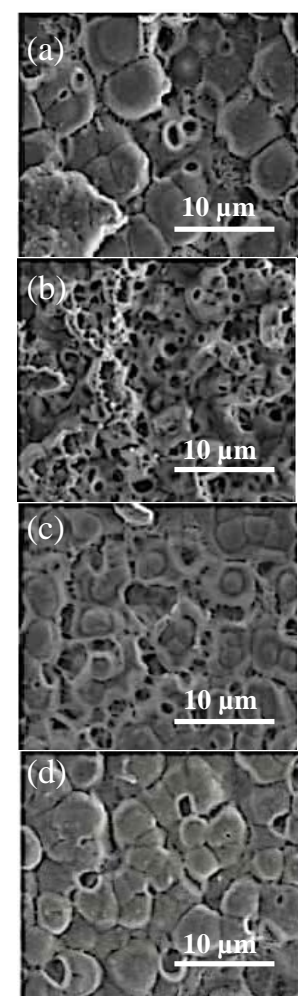

Fig. 1.SEM

Micrograph of surface Micrograph of surface view flat-sheet

membranes at 5000x magnification (a) pure PVDF (b)

PVDF/0.25NZ (c)

PVDF/0.50NZ (d) PVDF/0.75NZ

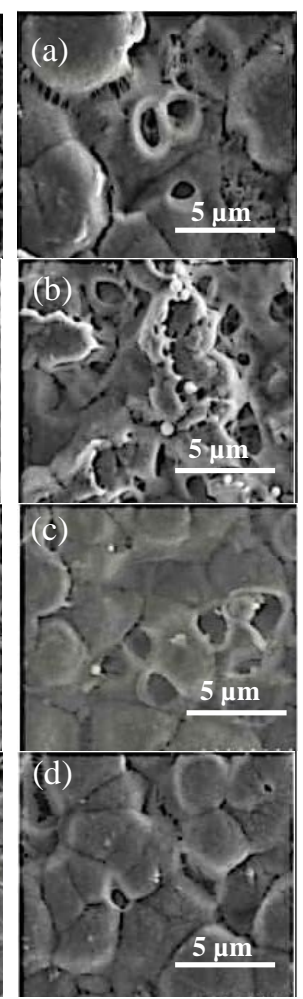

Fig. 2.SEM

view flat-sheet membranes at $10000 \mathrm{x}$ magnification (a) pure PVDF (b)

PVDF/0.25NZ (c) PVDF/0.50NZ (d) PVDF/0.75NZ 


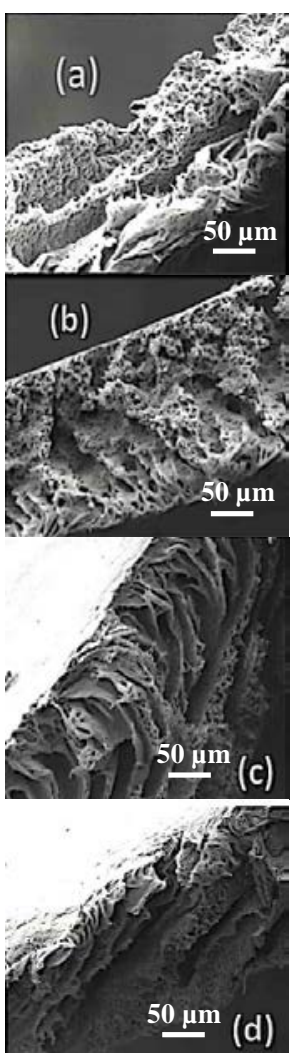

Fig. 3.SEM Micrograph of cross section view for (a)pure PVDF, (b) $\mathrm{PVDF} / 0.25 \mathrm{NZ}$, (c)PVDF/0.50NZ and (d) PVDF/0.75NZ membranes at $600 \mathrm{x}$ magnification

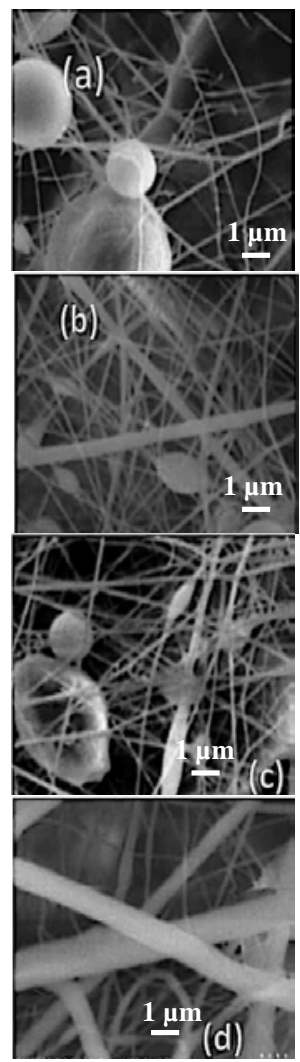

ig. 4.SEM images of nanofibers in electrospun membrane surface morphology of (a) Pure PVDF, (b) PVDF/0.25NZ, (c) PVDF/0.50NZ and (d) $\mathrm{PVDF} / 0.75 \mathrm{NZ}$ at $30000 \mathrm{x}$ magnification

\subsection{Contact angle}

In fig. 5 and fig. 6, due to the nature hydrophobicity of PVDF, the contact angle of all the fabricated membranes possessed a hydrophobic characteristic. The result showed that, the contact angle of flat-sheet and electrospun membranes was increased corresponding to the percent weight of nanozeolite. On the other hand, Kang et al., stated that the water absorption ability of zeolite particles is affected by fabrication methods [2]. Therefore, adding an extra amount of nanozeolite filler into PVDF polymer increase the contact angle, whereas the nanozeolite changed its hydrophilic nature to hydrophobic, similarly the different contact angle of the membrane carrier by different fabrication methods.

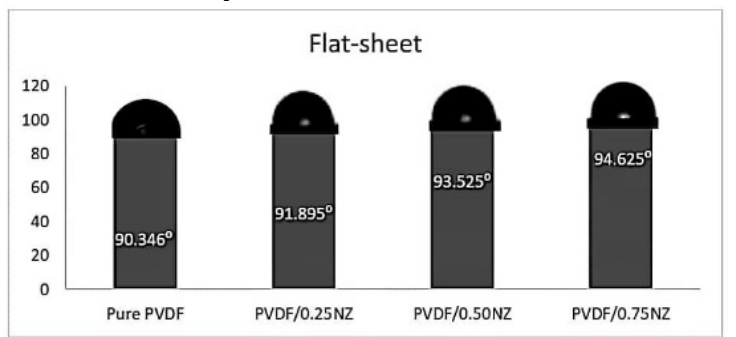

Fig.5.Contact angle of theflatsheet membranes

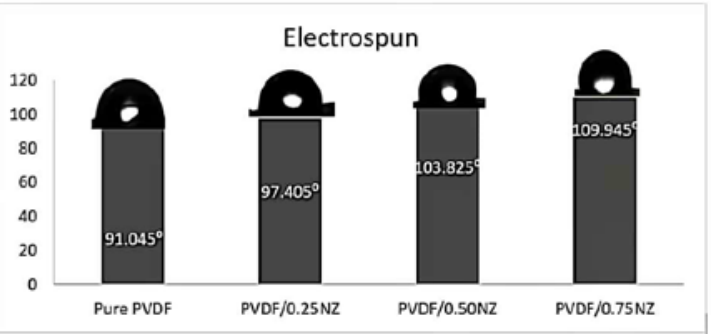

Fig.6.Contact angle of the electrospunmembranes

\subsection{Surface chemical composition}

As reported by Bai et al., the bond of 3022, 2980, 1403, 1185,878 and $840 \mathrm{~cm}^{-1}$ assigned to the vibration of PVDF [3]. Thanh et al., determining structure types of zeolites, the region of $400-1400 \mathrm{~cm}^{-1}$ is of interest.In flatsheet membrane (fig.7) with nanozeolite filler possessed an increasing intensityat $2921 \mathrm{~cm}^{-1}$ and $3023 \mathrm{~cm}^{-1}$, while electrospun membrane (fig. 8) the peak at $3024 \mathrm{~cm}^{-1}$ is slightly increased, those absorption peaks corresponded to C-H stretch. A shift peak position was occurred at the absorption peaks of $1667 \mathrm{~cm}^{-1}$ and $1671 \mathrm{~cm}^{-1}$ which are attribute to $\mathrm{C}=\mathrm{O}$ stretching. Both fabricated membranes also obtained similar an absorption peak at $1403 \mathrm{~cm}^{-1}$ which show the existence of $\mathrm{C}-\mathrm{H}$ bending alkanes group, however electrospun membranes showed a maximum absorption. While at peak $1071 \mathrm{~cm}^{-1}$ and $835 \mathrm{~cm}^{-1}$ were assigned to $\mathrm{C}-\mathrm{O}$ stretching and $\mathrm{C}=\mathrm{C}$ bending, both peaks indicate the incorporation of nanozeolite filler in polymer matrix.

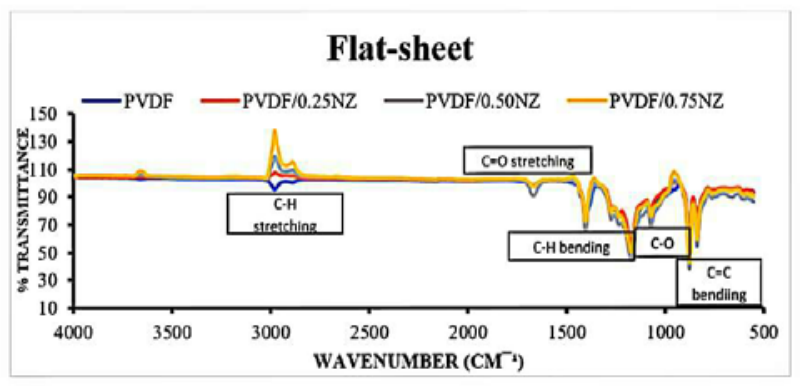

Fig.7.FTIR of flat-sheet membranes

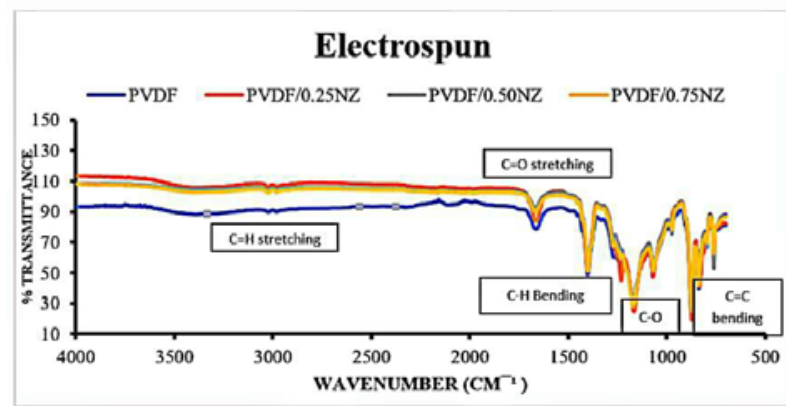

Fig.8.FTIR of electrospun membranes

\subsection{Mechanical Properties}

In fig. 9 and fig. 10, the higher tensile strength of the membrane was obtained by pure PVDF, while in young's modulus, a significant enhancement obtained from PVDF membrane incorporated with $0.25 \%$ of 
nanozeolite due to its higher interfacial area. For the decreasing of elongation in fig. 11 and fig. 12,lead to lower ductility of the membrane due to the rigidity of the membrane wherein the increased depth and width of finger-like voids as observed [4].Moreover, PVDF/0.25NZ obtained the higher tensile strength and elongation due to the alignment of fibers into polymer matrix, meanwhile PVDF/0.50NZ exhibited higher young's modulus where the membrane would become stiffer in this point due to disorder fibers on morphology.

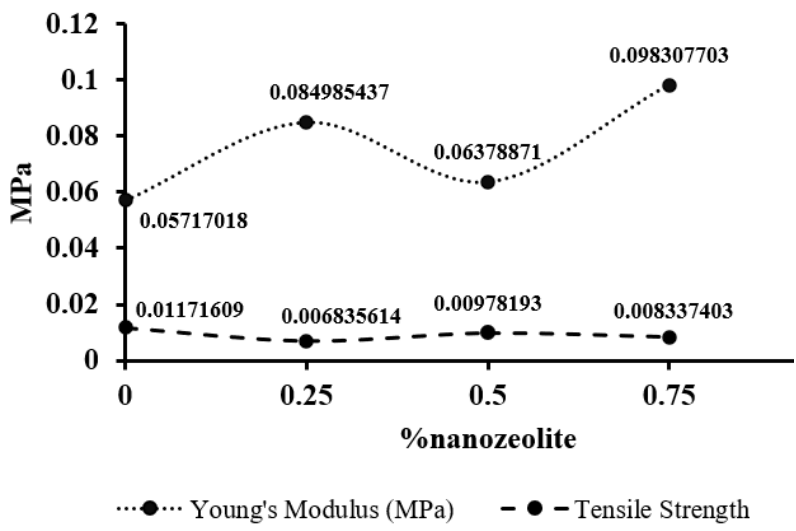

Fig.9.Tensile strength of the flat-sheet membranes

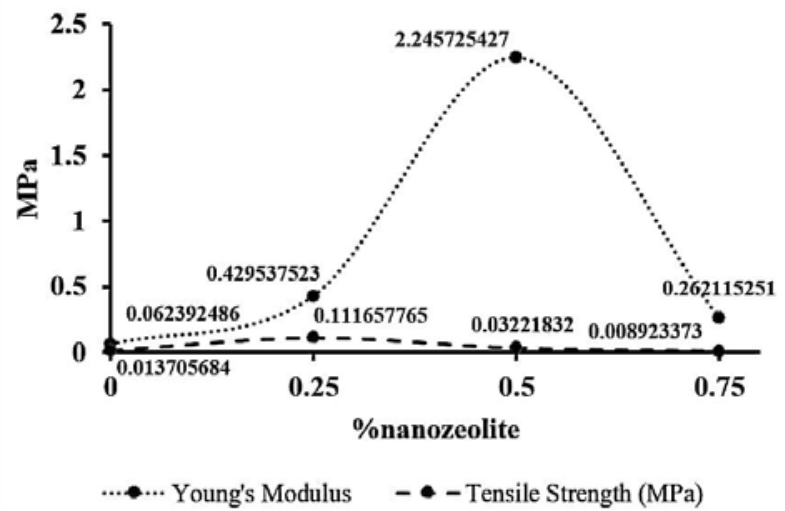

Fig.10.Tensile strength of the electrospun membranes

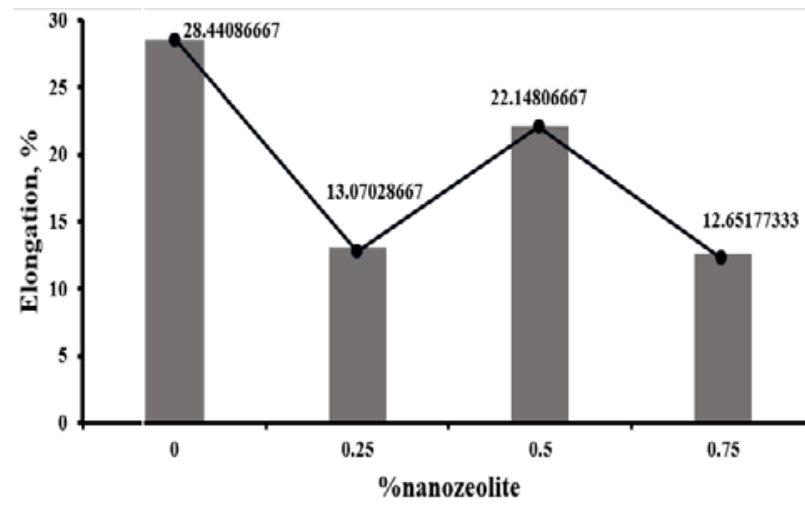

Fig. 11.\%elongation of the flat-sheetmembranes

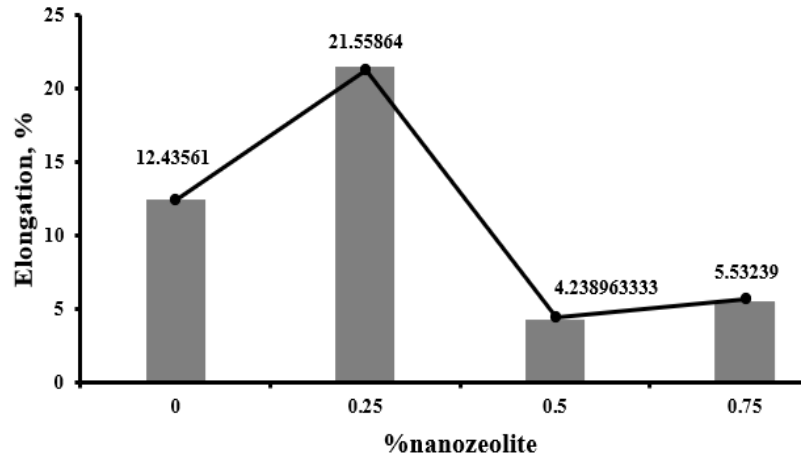

Fig.12.\%elongation of the electrospunmembranes

\section{Conclusion}

Nanocomposite fabrication of PVDF polymer with an additive of nanozeolite was successful fabricated and characterized. The addition of small amount of nanozeolite into polymer leaded a tremendous changed in morphology and structure for both flat-sheet and electrospun membranes, as increasing the amount of nanozeolite into PVDF polymer the flat-sheet resulted a decreasing in porous and electrospun obtained less beads and increasing in fiber diameter. Certainly, incorporation of PVDF polymer with nanozeolite in membrane was also confirmed by FTIR both fabricated membranes resulted same functional group.

An increasing hydrophobicity of the surface membrane were also obtained by both fabricated nanocomposite membranes whereas the nanozeolite filler changed its hydrophilic nature to hydrophobic, however the PVDF/0.75NZ of electrospun membrane showed a better hydrophobic surface with the highest contact angle of $109.945^{\circ}$ by the reason of high amount of nanozeolite and high absorption of alkanes. Moreover, in flat-sheet membrane the experimental result in tensile strength test showed that, the pure PVDF has a high tensile stress with $0.01171609 \mathrm{MPa}$ compared with the additional of nanozeolite, However, the PVDF/0.25NZ in electrospun membrane exhibit an optimum tensile strength with $0.111657765 \mathrm{MPa}$. Thus, PVDF membrane that fabricated by NIPS method has lower mechanical properties than the electrospinning method, further works is needed to investigate various nanozeolite loadings to understand its effect to the mechanical properties for both and tensile strength and elongation for NIPS. In contrast, in terms of young's modulus, the membrane in electrospinning technique is stiffer than NIPS.

Above all, in accordance of the result of these characterizations for both fabricated membranes, the fabricated nanocomposite of PVDF polymer with an additive of nanozeolite via electrospinning indicated as an excellent fabricated nanocomposite membrane.

\section{References}

1 H. Shi, F. Liu, and L. Xue, "Fabrication and characterization of antibacterial PVDF hollow fi bre membrane by doping Ag-loaded zeolites"J. 
Memb. Sci., vol. 437, pp. 205-215, (2013).

2 D. H. Kang and H. W. Kang, "Surface energy characteristics of zeolite embedded PVDF nanofiber films with electrospinning process"Appl. Surf. Sci., vol. 387, pp. 82-88, (2016).

3 H. Bai, X. Wang, Y. Zhou, and L. Zhang, "Preparation and characterization of poly(vinylidene fluoride) composite membranes blended with nano-crystalline cellulose"

4 C. Y. Lai, A. Groth, S. Gray, and M. Duke, "Nanocomposites for Improved Physical Durability of Porous PVDF Membranes” pp. 55-78, (2014) 